\title{
A Study on the Biological Control of Fusarium oxysporum Using Trichoderma spp., on Soil and Rockwool Substrates in Controlled Environment
}

\author{
Radu M. GIURGIU ${ }^{1 *}$, Adelina DUMITRAȘ ${ }^{2}$, Gavril MORAR ${ }^{1}$, \\ Petra SCHEEWE ${ }^{3}$, Fritz G. SCHRÖDER ${ }^{4}$ \\ ${ }^{1}$ University of Agricultural Sciences and Veterinary Medicine, Faculty of Agriculture, 3-5 Calea Mănăstur, Cluj-Napoca, \\ Romania; radu.giurgiu@outlook.com (*correspondingauthor) \\ ${ }^{2}$ University of Agricultural Sciences and Veterinary Medicine, Faculty of Horticulture, 3-5 Calea Mănăştur, Cluj-Napoca, Romania, \\ Romania;dumitrasadelina@gmail.com \\ ${ }^{3}$ University of Applied Sciences, Faculty of Agriculture/ Environment/Chemistry, Department of Fruit Growing and Tree Nursery, 2 Pillnitzer \\ Platz, Dresden, Germany; scheewe@htw-dresden.de \\ ${ }^{4}$ University of Applied Sciences, Faculty of Agriculture/ Environment/Chemistry, Department of Vegetable Growing, 2 Pillnitzer Platz, Dresden, \\ Germany; schroeder@htw-dresden.de
}

\begin{abstract}
Medicinal plant cultivation in Controlled Environment (CE) is increasing in the context of the new findings concerning the abiotic stress factors manipulation that leads to a significant increment in bioactive substances. Pathogen control is a vital part of the cultivation system, therefore the study was focused on biological methods of controlling a frequently occurring disease, by inoculating the sterile substrates specific in hydroponics, with the beneficial organism. Hypericum perforatum seedlings were inoculated with Fusarium oxysporum and with Trichoderma spp. The results showed that the method of inoculation had a significant effect on the success of the biological control. The plants treated with Trichoderma spp. had a two-fold increase in foliar biomass and better development of roots than the plants inoculated with the pathogen. Morphologically there were no significant differences, with some notable exceptions. The health status of the seedlings inoculated with the pathogen showed signs of the disease, even in the presence of Trichoderma spp. Finally, determinations of Volatile Organic Compounds (VOCs) at the root level showed that the biotic stress was considerable higher in the rockwool substrate that increased the secondary metabolism giving new perspectives in the cultivation of medicinal plants in CEA.
\end{abstract}

Keywords: food security, fungi, soilless, metabolites, St. John's Wort

\section{Introduction}

Controlled Environment Agriculture (CEA) has the advantage of precise manipulation of the environmental parameters that can determine high yields and quality of the crops (Chen et al., 2011). Using the knowledge of the plants together with the ever-developing technology, the products obtained can be improved for treating a large number of health conditions (Musharof, 2011).

The biochemical profile of the plants can indicate if there are any stress conditions. The secondary metabolism is not vital for the survival of the plants but it has a defensive role against pathogens that are responding to improper growing conditions (Hartmann, 2007; Atkinson and Urwin, 2012; Rejeb et al., 2014).

Soil-borne pathogens are some of the most daunting problems in crop protection. The fungi are difficult to observe, control and avoiding their occurrence is almost impossible. Fusarium spp. can damage up to 100 different species and can produce a series of toxic secondary metabolites that are a threat to the agriculture bio-safety, food security and the health of plants, animals, and people (Berges et al., 2013). F. oxysporum colonizes the plant through the root system, then the xylem, preventing the optimal development of the host plant (Gawehns et al., 2013). 
The biological control is gaining traction among farmers and researchers because of its efficiency and have less negative impact on the environment. Furthermore, this method is important in cultivating medicinal plants where studies showed that the reaction of the plants to the pathogens can increase the activity of the secondary metabolism, their root systems acting as a chemical factory (Hadi et al., 2013; Ding et al., 2014; Ganwar et al., 2014; Bahraminejad et al., 2015).

In the hydroponic systems, the pathogen spreads rapidly and the exposure of the roots in the nutrient solution can spark a quick loss of the crops. The fungi can develop in the sterile substrates also, if the conditions allow because it can be carried in the growing chamber through air spores or by the growers, through the equipment (Postma et al., 2013).

One of the most popular microorganisms for biocontrol is Trichoderma spp., arbuscular fungi that can be found in different types of soil. In nature, the fungi can be hardly identified due to its low concentrations (Cornejo et al., 2015). Trichoderma spp. have a symbiotically relation with the roots of the plants, helping the nutrient flux and the development of the plants (Althaf and Srinivas, 2013). Trichoderma spp. are natural competitors of some pathogens like Fusarium, Pythium, etc., competing with them for the nutrients, space and also through mycoparasitism (Lace et al., 2014). The method of inoculation is very important for the success of the biological control strategy and can differ in regard with different substrates (El Komy et al., 2015).

The inoculation of the substrates with Trichoderma spp. has shown numerous positive results, but for hydroponics, the application method has not been studied enough, yet. New studies proved that the biological control can stimulate the secondary metabolism resulting in higher concentrations of bioactive substances (Abdelrahman et al., 2016).

Most research is focused on the application of Trichoderma spp. to the conventional soil cultivation and the results in higher plant biomass, controlling the pathogen or improving the rooting systems of the plants (Ming et al., 2013). The latest studies discovered an impact of the beneficial microorganism for stimulating the secondary metabolism, therefore improving the bioactive substances content of the plants (Lopez-Bucio et al., 2015). These works are advancing the scientific knowledge and are developing growing protocols that will aim to improve the quality, not just the yield of the crops cultivated in a controlled environment.

The aim of this study was to investigate how Trichoderma spp. can be applied on sterile substrates compared to soil and the efficiency of the product to control F. oxysporum in the seedlings of Hypericum perforatum. The study follows different inoculation methods opening new discussions in the field of CEA and biological control of the pathogens.

\section{Materials and Methods}

\section{Biologicalmaterial}

The seedlings were obtained in the same manner for the two inoculation methods. The plant used in the experiments was Hypericum perforatum (HA336, Jelitto, Germany) obtained from seeds. The plants were seeded in alveolar trays in soil substrate (C200 with 20\% sand). The trays were covered with a black textile for the germination process. After germination, the trays were uncovered but kept under a transparent dome that kept high humidity and temperature for the first steps in the development of the seedlings. After the appearance of the first pairs of true leaves, the domes were removed and the seedlings were monitored further. Approximately 30 days have passed from seed to the time of which the seedlings were ready for the experiment.

Preparation of Fusarium oxysporum and Trichoderma spp. inoculation solutions

For the both inoculation series, the solutions were made following the same method. The application protocol differed and further explained in the methods section.

The Trichoderma inoculation solution was prepared using a commercial soluble powder which contains Trichoderma harzianum $\left(2 \times 10^{-7} \mathrm{CFUg}^{-1}\right)$ and Trichoderma konigii $(3 \times$ $10^{-7} \mathrm{CFUg}^{-1}$ ) (Promot WP, from Katz Biotech AG, Germany). The inoculation solution was obtained by mixing $1 \mathrm{~kg}$ product in $100 \mathrm{~L}$ of water and left half an hour for a homogenous dissolution.

The F. oxysporum was isolated from infected strawberries (Fragaria spp.) at the Institute for Breeding Research on Fruit Crops (Julius Kühn Institute) Dresden, Germany. The fungus was cultivated on potato dextrose agar (Fig. 1) and incubated at the University of Applied Sciences from Dresden, at $20^{\circ} \mathrm{C}$, in darkness. $F$. oxysporum was replicated every 4-8 weeks, depending on the research project's demand. In order to prepare the inoculation solution, the petri dishes were collected and the development of the fungus was determined. Each probe was mixed with distilled water and observed under the microscope, counting the conidia. The concentrated solution $\left(10^{7} \mathrm{CFUg}^{-1}\right)$ was mixed with water $1: 100$ in order to have the same concentration as the Trichoderma spp. solution (Fig. 2).

\section{First inoculation: at the substrate level}

Before the inoculation process, the substrate was dehydrated (not irrigated for a few cycles) for the media to take in the solution in a high degree. Each substrate contained four even Hypericum perforatum seedlings. The root system was well visible at the bottom of the substrates. Two centimetres of the media were introduced in the inoculation solutions and left an hour for full absorbance. For the first experimental series, six variants were prepared in a single media, the soil (C200 with $20 \%$ sand). The experiment was designed in randomized blocks. The six variants were the following:
A) not treated (control);
B) inoculated with F. oxysporum;
C) inoculated with Trichoderma spp.;
D) inoculated with $F$. oxysporum, after one hour, inoculated with Trichoderma spp.;

E) inoculated with Trichoderma spp., after one hour, inoculated with F. oxysporum;

F) inoculated with $F$. oxysporum and with Trichoderma spp., in the same time.

The last three variants were chosen to see if the time of the Trichoderma application (prevention, control) is relevant in the 
262

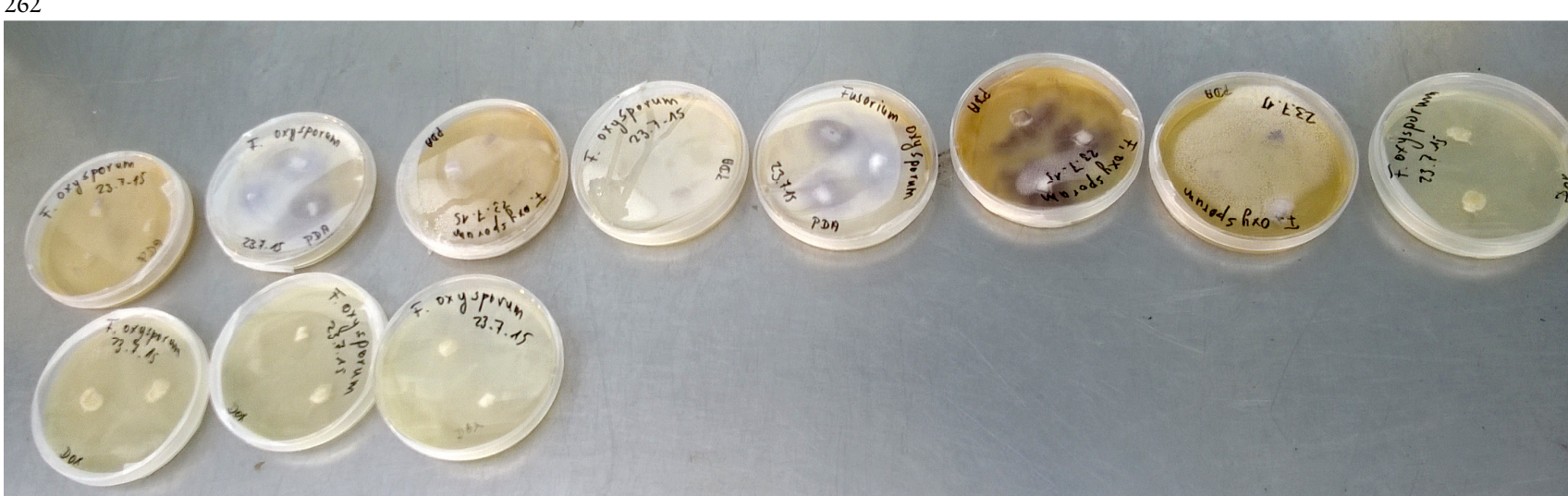

Fig. 1. Fusarium oxysporum cultivated for the experiments in different media

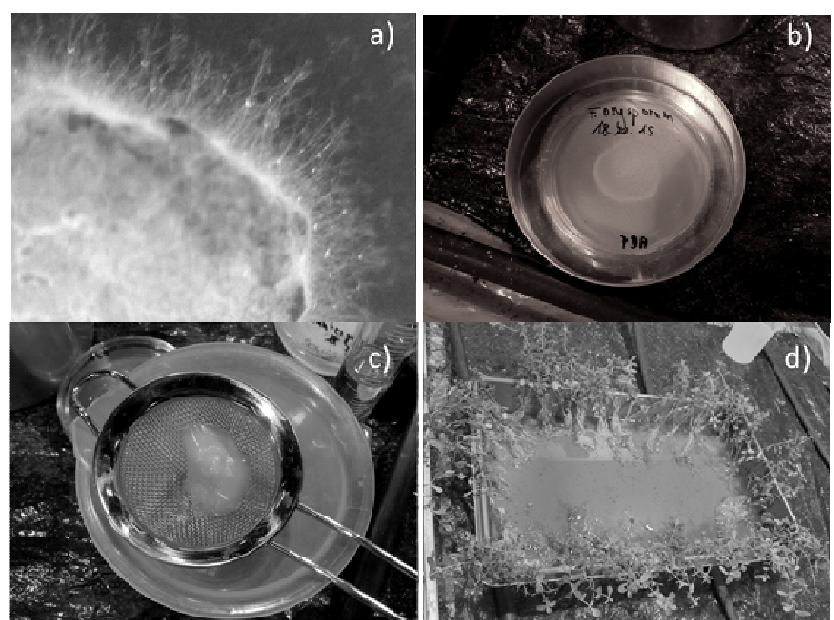

Fig. 2. Preparation of the Fusarium oxysporum solution for the inoculation of Hypericum perforatum seedling; a) microscope image of F. oxysporum; b) F. oxysporum on AGAR media; c) preparation of the concentrate solution through filtration; $d$ ) the inoculation of the $H$. perforatum seedlings

biological control strategy. After the inoculation process, the seedlings were transplanted into pots with the same soil mix, having a diameter of $11 \mathrm{~cm}$ and an approximate volume of 500 $\mathrm{cm}^{3}$.

\section{Second inoculation: direct root inoculation}

For the second series, the seedlings were obtained in the same way as described before. The soil was removed from the roots by a water jet. The seedlings were kept in the water for the entire process to protect them against dehydration. The inoculation solutions were obtained in the same way as described in the first inoculation series. Similar looking seedlings were selected and their roots were introduced in the prepared inoculation solutions. For this series, four variants were designed, as such:
A) not treated (control);
B) inoculated with F. oxysporum;
C) inoculated with Trichoderma spp.;
D) inoculated with $F$. oxysporum and with Trichoderma spp. at the same time.

The plants were left one hour to take in the solution, then randomly selected and moved into $11 \mathrm{~cm}$ diameter pots with soil (C200 with $20 \%$ sand), as well as in rockwool cubes $(7 \times 7$ $\times 10 \mathrm{~cm}$ ). The two media had similar volume/ available space for root development. In the soilless substrate, the root system of the seedling was covered with sand for the contact with the substrate and to avoid oxidation.

\section{Experimentaldesign}

The plants were set in randomized blocks, each variant having 20 substrate cubes per variant with 4 seedlings per cube $(\mathrm{N}=20 \times 6 \times 4=480)$, for the first series and 10 cubes per variant per substrate type (soil and rockwool) $(\mathrm{N}=10 \times 4 \times 2$ $=80$ ). The environmental parameters of the grow chamber were set as following: temperature $21 \pm 2{ }^{\circ} \mathrm{C}$, relative air humidity $60 \pm 15 \%$, automated irrigation correlated with the solar radiation applied daily, $15 \mathrm{~min}$ intervals. For the plants grown in the rockwool, a light nutrient solution was applied to match the nutrients available in the soil. The nutrient solution was made with mixing Universal Blue concentrate with water, reaching an electrical conductivity (EC) of $0.6 \mathrm{mS}$.

\section{Observation and measurements conducted}

From the inoculation moment until the harvest, the plants were monitored daily. The success of seedling transplant was observed, marking the plant mortality and plant health parameters, graded from 1 to 5 (with highest grade 5). The symptoms induced by the pathogen as described in the scientific literature were monitored. The plant development was observed through the measurements of plant morphological traits such as stem length, leaf area, internodes number and length, branching process etc.

At the harvest moment, the biomass was measured(upper and roots). In the second series of the inoculation, every two weeks the tips of the plants $(1 / 3)$ were cut to stimulate branching that was later monitored to see if there are any significant differences among the variants in regard with the new stem growths.

\section{Chemical analyses}

Solid phase micro extraction (SPME) is a sample preparation technique without solvents, used for collecting Volatile Organic Compounds (VOCs) under static conditions, where volatiles are adsorbed on a fiber in Headspace (HS). HS is the gas space above the sample in a closed vial. For a collection of volatiles by SPME it is desired an equilibrium of analytes between the SPME fiber, the releasing source, and the HS. 


\section{HS- SPME extraction}

In each vial (bag) 3 grams of Hypericum perforatum dried seedling roots were placed. Then the fiber attachment needle was inserted and SPME fiber was exposed in the headspace for $30 \mathrm{~min}$ during collection/adsorption. At the end of time, the fiber was retracted, holder with the saturated fiber was analyzed by injection in GC injection port. The fiber used was Carboxen / PDMS $75 \mu \mathrm{m}$. Before used, the fiber was preconditioned at $300{ }^{\circ} \mathrm{C}$ during 60 minutes in the GC injector port.

\section{$G C-M S$ analysis}

GC-MS used for analysis was Agilent 7890 \& 5975 Series MSD. For qualitative determination, the MS system was operated in SCAN mode. The carrier gas was helium at a

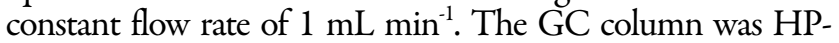
$5 \mathrm{MS}(30 \mathrm{~m}, 0.25 \mathrm{~mm}, 0.25 \mu \mathrm{M})$, with the initial temperature of the oven at $60{ }^{\circ} \mathrm{C}$, initial time: $1 \mathrm{~min}$, then gradient $30^{\circ} \mathrm{C}$ $\min ^{-1}$ to $150{ }^{\circ} \mathrm{C}$, then gradient $30{ }^{\circ} \mathrm{C} \min ^{-1}$ at $250{ }^{\circ} \mathrm{C}$ and maintained at $250{ }^{\circ} \mathrm{C}$ for $5 \mathrm{~min}$. The identification of compounds was made by using databases: NIST L14.

\section{Statistical methods}

All the statistic calculations and graphics were realized using the IBM SPSS software. The graphs were edited and refined using the Adobe Illustrator Software. The means for each variant were compared using ANOVA (mono or bi factorial), validating the Levene test which showed the homogeneity of the variants. Afterward, the $t$ (Student), Tukey, Dunnet or Games-Howel tests were used to determine the significance of the differences between the means $(p<0.05)$. In determining the significance of the differences between the mortality of the seedlings in different variants, the Tarone-Ware test was applied, calculating $\mathrm{Chi}^{2}$ and the functions were represented graphically.

\section{Results and Discussion}

First inoculation: at the substrate level

Although easy to interpret statistically, the results of the first experimental setup were difficult to track back to the causes because of the conflictual and contra-intuitive figures. Generally, where both the inoculation solutions were present, the plants had the worst development, and the ones not treated (control) had the best one. Nieto-Jacobo $e t$ al. (2017) have shown that Trichoderma strains are dependent on many variables and are affected by the environment, which can lead to negative results. Therefore, we can assume that the interaction of Trichoderma spp. with the pathogen created an environment in which the plant had detrimental development. The findings of Jelinski et al. (2017) add to this theory through the fact that the pathogenesis of the $F$. oxysporum populations is a complex process and what happens at the root level should be thoroughly studied.

The untreated plants had the longest stem length and had merely shorter roots than the seedlings that were inoculated just with Trichoderma spp. which was in accordance with previous studies (Krupa et al., 2016), but not statistically significant. The plants inoculated with Trichoderma spp. had longer internodes than the untreated or infected ones (Fig. 3). Bona et al. (2017) have shown that the presence of fungus is correlated with longer internodes, but why this phenomenon was recorded is still unknown as the majority of studies correlate length of the internodes with light availability (Kahlen and Strützel, 2011); for this project, all plants had the same light conditions.

The plants inoculated with Trichoderma spp. and those not treated (control) had significantly larger biomass than all the other variants in which Fusarium was present, which is in accordance with the previous studies (Ommati and Zaker, 2012; Martinez et al., 2015; Lecomte et al., 2016) (Fig. 4). The three variants in which Fusarium was present showed no significant differences compared to each other. The root system biomass was the highest for the untreated plants (control) followed by those inoculated with Trichoderma and then the ones inoculated with Fusarium. The plants inoculated with both solutions in the same time had the smallest roots. It is worth mentioning that the plants attacked by the pathogen are triggering the secondary metabolism and so responding to the threat (Zhang et al., 2017), so the plants inoculated with $F$. oxysporum, might have successfully managed to fight back the pathogen, resulting in no statistical differences (Fig. 5). Other studies on phytohormones add to this idea, concluding that in some cases, the presence of $F$. oxysporum actually improves the growth of the plant (Di et al., 2016).

But this could be better understood through the following mortality function that was calculated in regard to the transplantation success and the effect of the solution on this process (Fig. 6). It was observed that the plants inoculated with the Trichoderma spp. had the highest mortality, reaching an index of 0.5 before harvest. $(1=$ successful transplant, $0=$ dead seedling. In the other variants, the rate of mortality was lower. This finding is in contrast with previous studies that claim that the beneficial organism is actually boosting the seedling development (Lingyun et al., 2017). Other authors discussed the paradoxical mutualistic-parasitic effect of Trichoderma spp. (Hajieghrari and Mohammandi, 2016) and so we could conclude that in this trial the beneficial organism acted as an inhibitor of the seedling transplantation and not as helping through the process. It might have been that later on, in the vegetation cycle the surviving seedlings would prosper on the beneficial organism, but this has to be studied in the future research projects.

\section{Second inoculation: direct root inoculation}

In the first trial, there were no significant differences between the three variants in which Fusarium and Trichoderma were used in different time combinations, therefore these three variants were simplified to one in which both inoculation solutions were added simultaneously as described by Ommati and Zaker (2012).

In the second trial, the differences between the variants were more clear and in accordance with the previous studies (Elsharkawy et al., 2013). The second set of experiments was original by studying rockwool as a substrate, compared with the results on the conventional soil cultivation. George (2014) showed in experiments on Tomato plants grown hydroponically that the fungus occurrence is significant even if there is a soil-free medium. Other studies showed the difficulty with the mycorrhizal colonization in rockwool and the subtle hints of their success shown in the total 
264

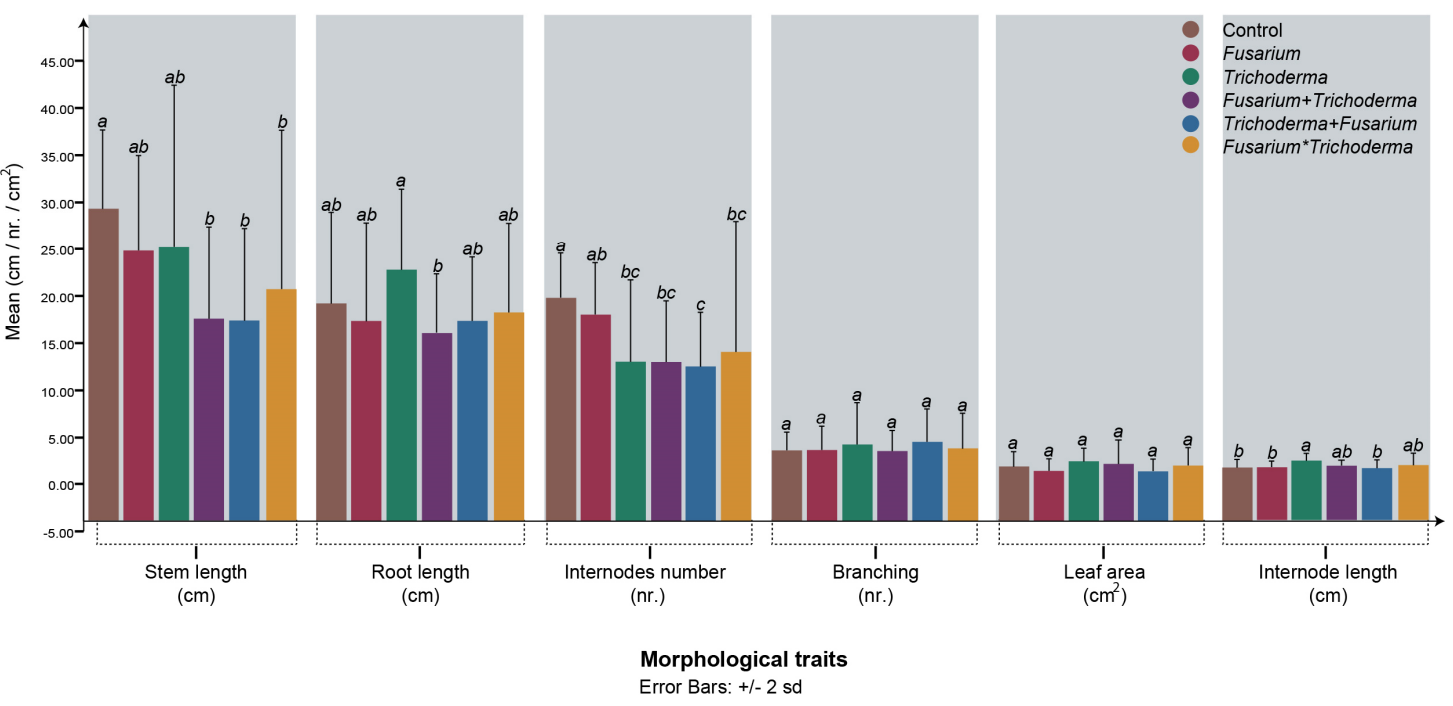

Fig. 3. Measurements of the morphological traits of Hypericum perforatum under different treatments, after the first trial (the different letters represent significant differences; $\mathrm{p}<0.05$ )
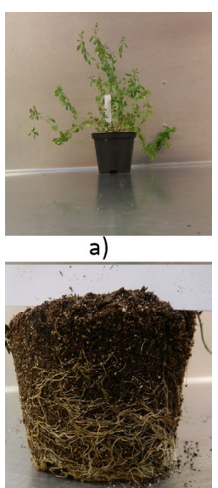

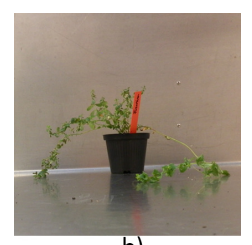

b)

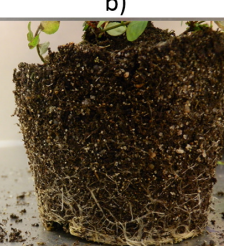

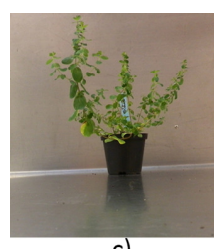

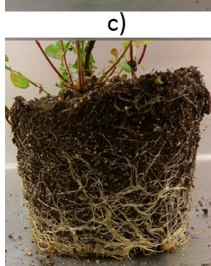

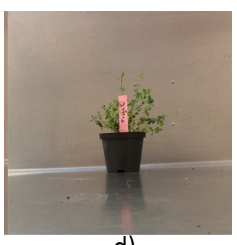

d)

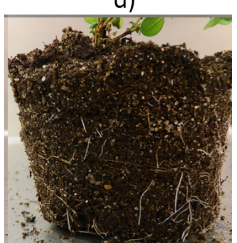

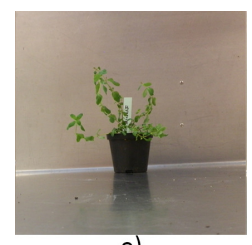

e)

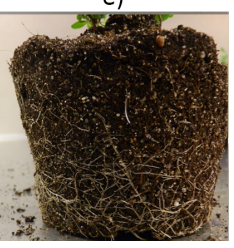

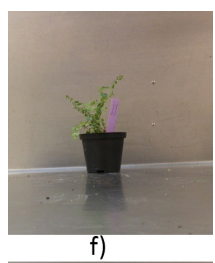

f)

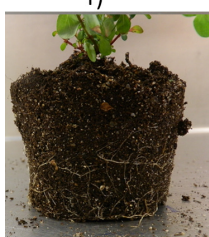

Fig. 4. Hypericum perforatum: a) control (without inoculation); b) Fusarium oxysporum inoculation; c) Trichoderma spp. inoculation; d) Fusarium oxysporum inoculation, after one hour Trichoderma spp. inoculation; e) Trichoderma spp. inoculation, after one hour Fusarium oxysporum inoculation; f) Trichoderma spp. inoculation and Fusarium oxysporum inoculation, at the same time

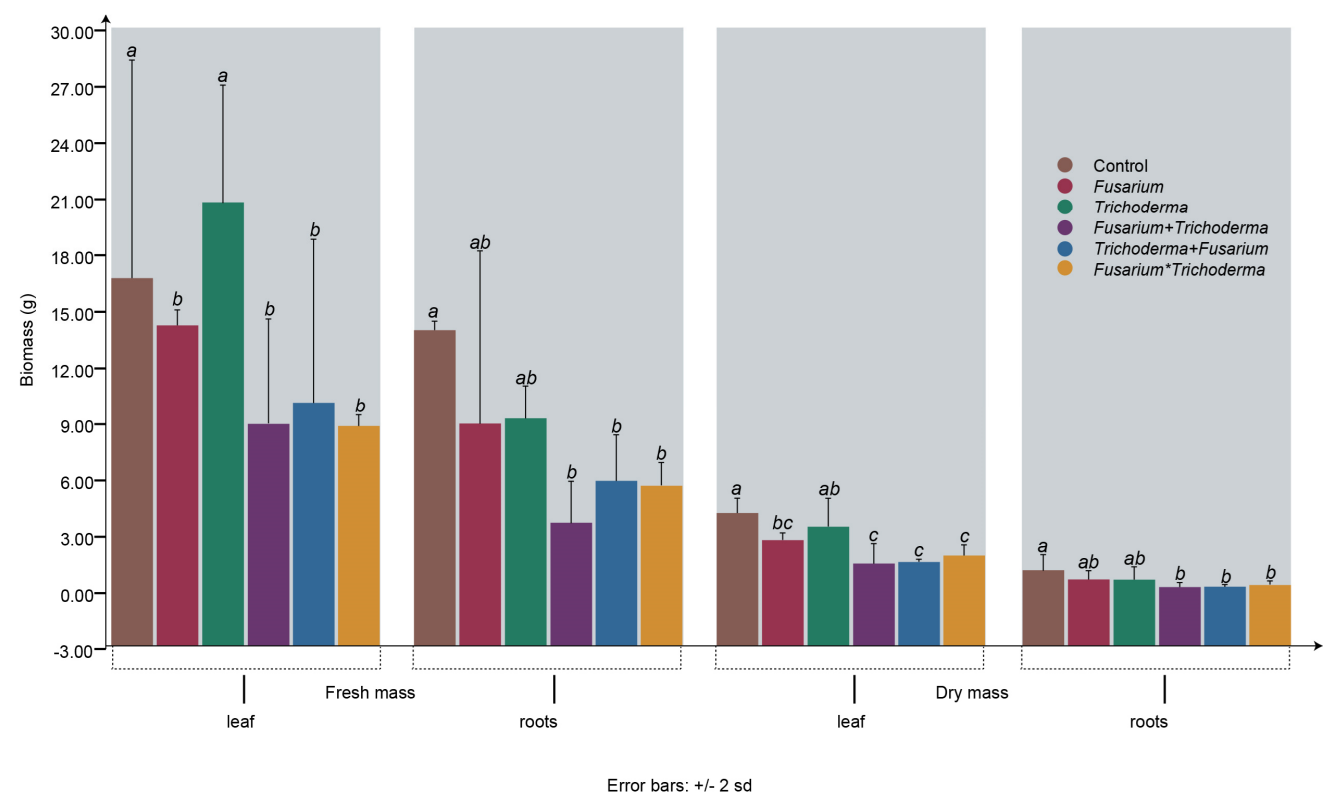

Fig. 5. Hypericum perforatum biomass in different treatments (the different letters represent significant differences; $\mathrm{p}<0.05$ ) 
Seedling Mortality Functions

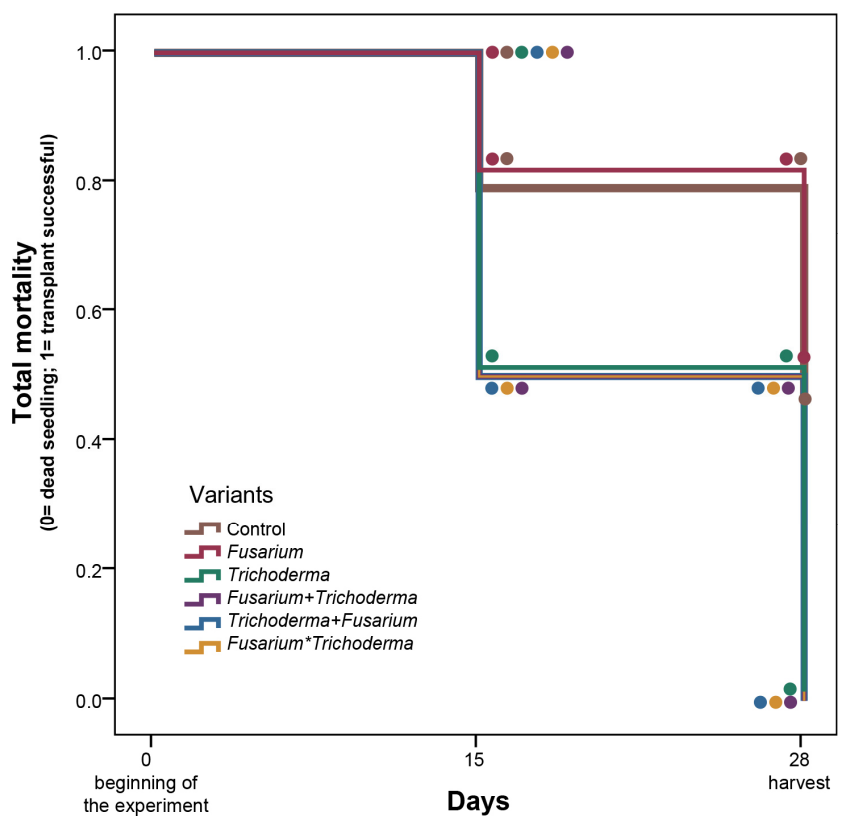

Fig. 6. Seedling mortality functions from the transplantation of the seedlings to the harvest

sugars content, but not in the growth parameters (Michałojć et al., 2015). In the present context of biological control of pests and diseases in CEA, it is important to come up with protocols for successfully using beneficial organism to colonize the substrates. The results shown in this manuscript are describing the inoculation of the seedlings. The root microbiome might need more time to stabilize so the future research should focus on the entire vegetation cycle and careful selection of strains that would be more applicable for soilless cultivation (Sheridan et al., 2017).

Regarding some morphological traits, the plants cultivated in soil were significantly longer than the plants cultivated in rockwool, but there were no significant differences between the inoculation applications (Fig. 7). The reason for the differences between the plants grown in soil and the ones grew in rockwool could be that the sterile substrate acts as an inhibitor of the substances inoculated as reported by Best et al. (2014).

The other morphological features that were measured had little to no statistically significant differences. It is worth to reiterate that the effect of the pathogen or the beneficial organism could be very subtle and could be only observed later on (Sheridan et al., 2017), but for reasons of high risk of additional factors such as nutrient solution, irrigation frequency etc., the experiment was terminated at the seedling phase.

For the plants cultivated in rockwool, there were no measurements of root biomass due to the difficulty of nondestructive measurements methods. The plants cultivated in soil had higher biomass compared with the ones cultivated in rockwool. Rubio et al. (2017) proved that the NPK fertilization can have a conflicting effect on the plant development in relation with the Trichoderma spp. Therefore, although the fertilization was made to match the available nutrients from the soil, the plants in rockwool might have had this disadvantage. As it happened in the first set of experiments, the smallest figures were registered for the plants inoculated with both solutions in the same time, in accordance with previous studies (Martinez et al., 2015). This fact can highlight the need for preventive treatment with Trichoderma and healthy microbial communities' establishment before the occurrence of the pathogen. The same aspects could be noted in regard with the root system biomass, with the untreated plants having the highest fresh biomass followed by those inoculated with Trichoderma spp and $F$. oxysporum The plants treated with both solutions showed the smallest root biomass (Fig. 8).

After drying, the results showed a more significant difference, the beneficial organism having a positive effect on the plant biomass. The plants inoculated with Trichoderma spp. and those not treated had significantly larger biomass in comparison with the other variants. An interesting observation was that where both the pathogen and the Trichoderma spp. were present, the biomass was bigger than where plants were inoculated solely with Fusarium, showing that the beneficial microorganism has a positive effect on the development of the plants (Fig. 9).

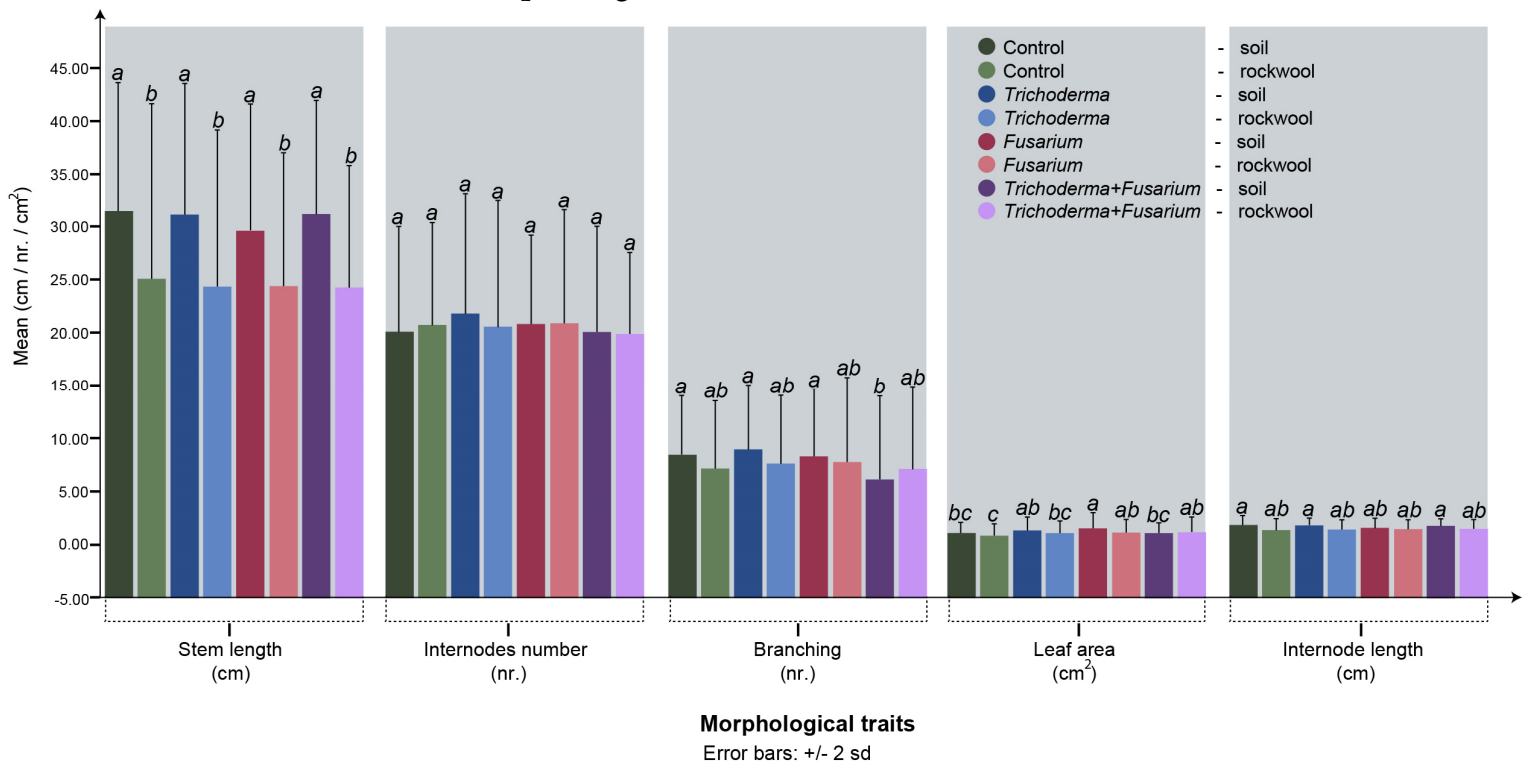

Fig. 7. Measurements of the morphological traits of Hypericum perforatum under different treatments, after the first trial (the different letters represent significant differences; $\mathrm{p}<0.05$ ) 
A)

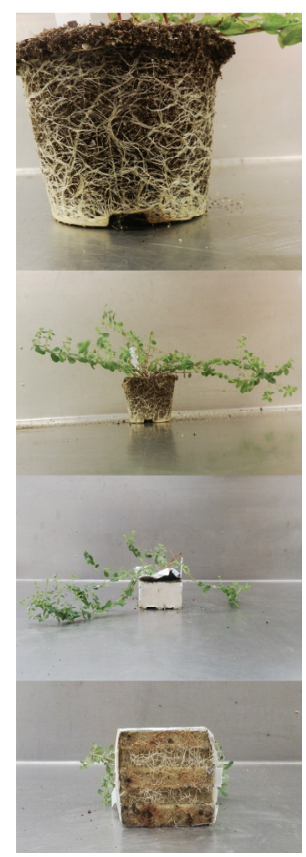

B)

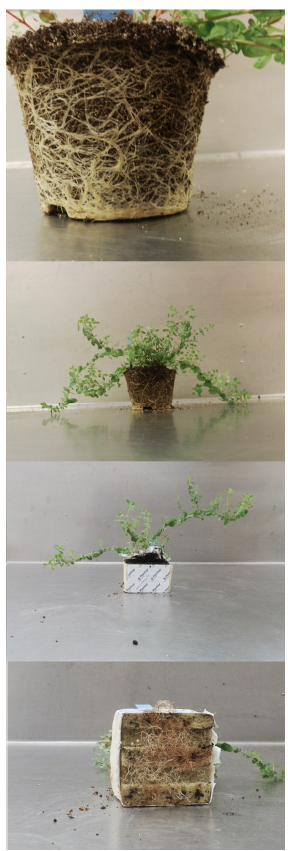

C)

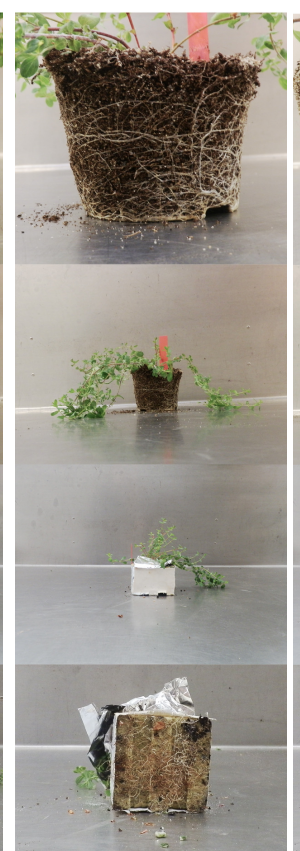

D)

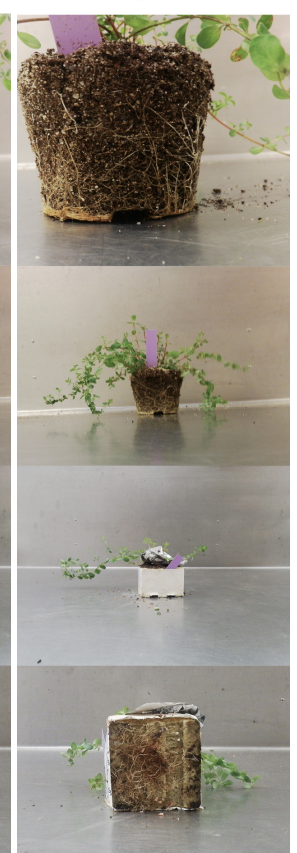

Fig. 8. Hypericum perforatum with different inoculation solutions: A) control (without inoculation); B) Fusarium oxysporum inoculation; C) Trichoderma spp. inoculation; D) Trichoderma spp. inoculation and Fusaiurm oxysporum inoculation, in the same time; the variants in rockwool and soil

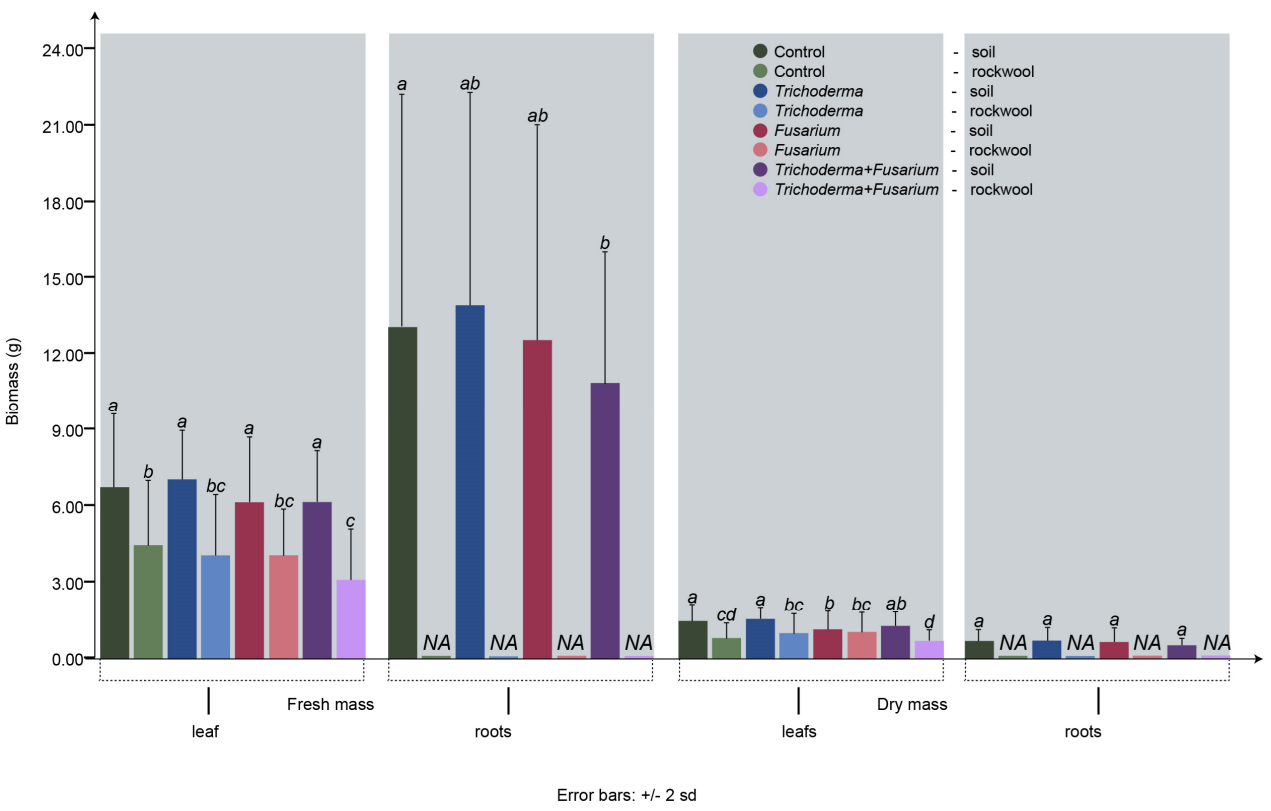

Fig. 9. Hypericum perforatum biomass in different treatments (the different letters represent significant differences; $\mathrm{p}<0.05$ )

The plants cultivated in rockwool did not show differences in biomass as the ones in the soil, showing that the biological control could be less effective for the sterile substrates. This highlights the importance of future research projects that will aim to develop protocols of applying biological control for pests and diseases in sterile substrates (Rubio et al., 2017; Sheridan et al., 2017).

The mortality distribution functions were calculated as described in the previous trial. The Tarone-Ware test was used but no significant differences were observed ( $p>0.05 ;$ chi $^{2}=$ $12.695)$ all the variants having a survival index above $0.8(1=$ successful transplant, $0=$ dead seedling). This can be a good method of inoculation, directly on the roots, before transplanting to the final culture.

During the experiment, the health state of the plants was evaluated and graded on a 1 to 5 scale with 5 being very healthy (Fig. 10). All the plants started on the level 5, as randomly selected from the inoculated seedlings. The plants inoculated with Trichoderma registered a sudden health state degraded explained by a possible stress in the inoculation/transplantation process, but later having an increase in the health state that has put the variant in first place in terms of health dynamics. The rest of the variants had a clear decrease of health state at the harvest moment, but it was not a linear progression. 


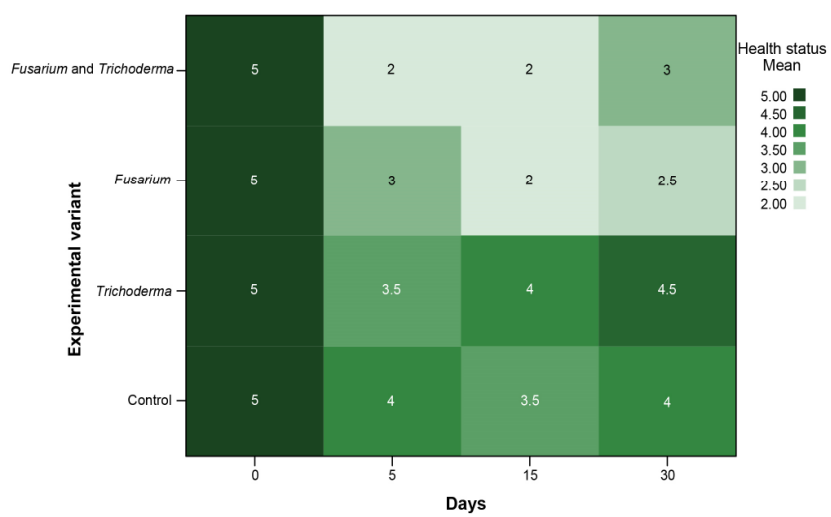

Fig. 10. Plant health state in the experimental variants, throughout the experiment, noted from 1 to $5(5=$ perfect health state, 1 = poor health state)

It has been observed that at the mid of the experiment (between the start and harvest of the seedling) the plants had the lowest health status. The plants inoculated with Fusarium had finally the lowest rating, while the plants inoculated with both of the solutions in the same time had an impressive recovery in the last stage of the experiment. This can be correlated with the fact that the Trichoderma had a positive effect, in accordance with previous findings (Sundaramoorthy and Balabaskar, 2013).
Metabolite activity at the root systems

For the second inoculation experiment, some chemical analyses of the roots were conducted in order to assess the differences between the variants in regard to the bioactive substances. There were a significant higher number of metabolites identified for the plants cultivated in rockwool compared with the ones grown in soil. The last category, only the Cyclopentasiloxane was identified with a high degree of confidence. The plants grown in rockwool had a larger spectrum of bioactive substances such as Benzofuranonetetrahydro-trimethyl, Trimethyl-methyleneoctahydromethanoazule, alpha-pinene, Cyclopentasiloxane, and others that were not as often in all the treatments (Fig. 11). This fact highlights an interesting idea in which the sterile substrates, which can be more difficult to manage than soil, can lead to a higher metabolite activity due to the stress factors. Kowalczyk et al. (2016) showed that there are no significant differences between the different soilless media that can be used in hydroponics, in regard to the nutritional content of the plants. And in general, the differences of the plant's quality are correlated with the cultivar rather than the agricultural system (conventional or soil) (López et al., 2014), so the mere stress degree at the root system because of the more difficult to manage rockwool, could have improved the bioactivity of the roots. These findings should be further studied as they pen interesting opportunities for the modern agricultural systems,
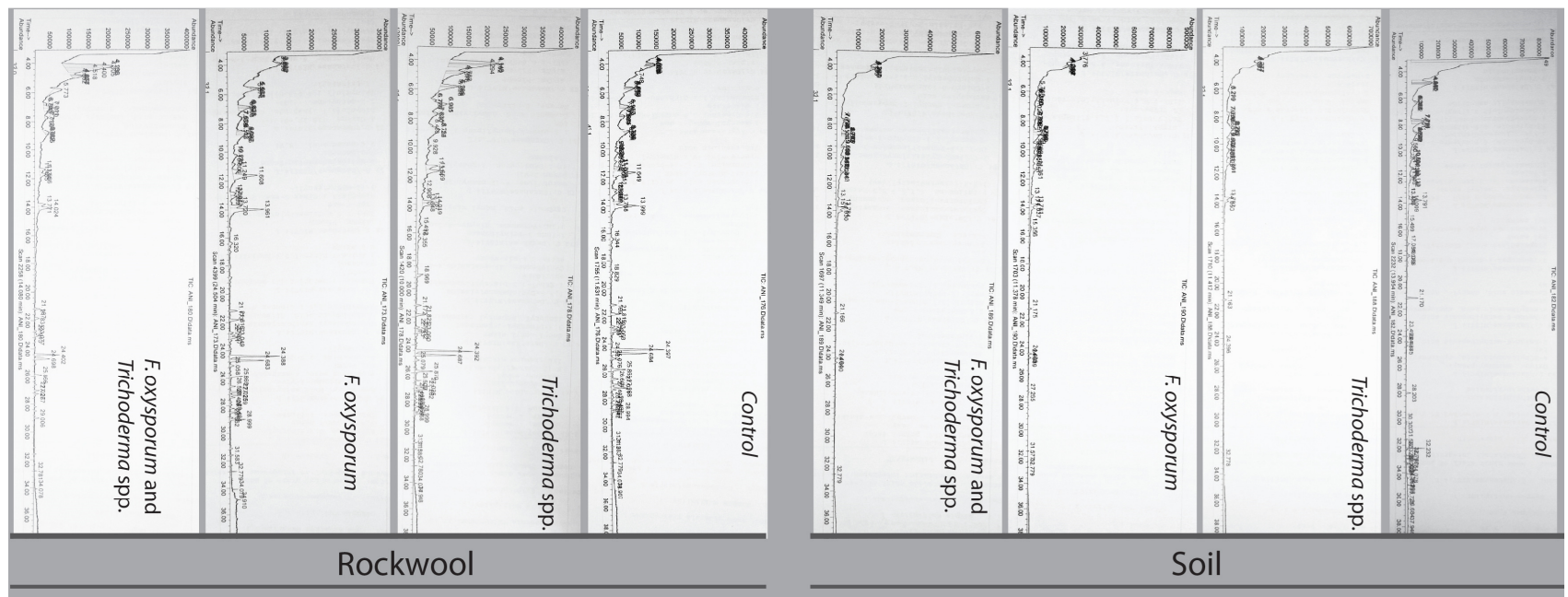

VOC Metabolites identified at the root level of the plants

\begin{tabular}{|c|c|c|c|c|c|c|c|c|}
\hline Substrate & Treatment/Metabolite & Cyclopentasiloxane & Limonene & alpha-pinene & Naphthalene & endo-Borneol & Tricyclo undec-9-ene & Bezofuranone \\
\hline \multirow[t]{4}{*}{ Soil } & Control & $x$ & $x$ & & & & & \\
\hline & F. oxysporum & $\mathrm{x}$ & $x$ & & & & & \\
\hline & Trichoderma & $\mathrm{x}$ & & & & & & \\
\hline & F.o. and T. spp. & $x$ & & & & & & \\
\hline \multirow[t]{4}{*}{ Rockwool } & Control & $x$ & & $x$ & $x$ & $x$ & & $x$ \\
\hline & F. oxysporum & $x$ & & $x$ & $x$ & $x$ & $x$ & $x$ \\
\hline & Trichoderma & $x$ & & $x$ & $x$ & $x$ & & $x$ \\
\hline & F.o. and T. spp. & $x$ & & $x$ & & & & $x$ \\
\hline Substrate & Treatment/Metabolite & Byciclohexane & Trymetil-6-methyleneoctahydro & Bicycloheptan-2-c & Copaene & Bis-phenol & alpha-ylangene & beta-pinene \\
\hline \multirow[t]{4}{*}{ Soil } & Control & & & & & & & \\
\hline & F. oxysporum & & & & & & & \\
\hline & Trichoderma & & & & & & & \\
\hline & \begin{tabular}{|l} 
F.o. and T. spp. \\
\end{tabular} & & & & & & & \\
\hline \multirow[t]{4}{*}{ Rockwool } & Control & & $x$ & & $x$ & $x$ & $x$ & \\
\hline & F. oxysporum & $x$ & $x$ & $x$ & $x$ & & & \\
\hline & Trichoderma & & $x$ & & $x$ & $x$ & $x$ & \\
\hline & F.o. and T. spp. & & $x$ & & & & & $x$ \\
\hline
\end{tabular}

Fig. 11. Graphs showing the differences of the metabolic activity at the root level of the plants. The table shows the VOCs identified in the different treatments 
268

based on total environmental control and big data sets (Tremblay, 2017).

\section{Conclusions}

The study showed that the method of inoculation with beneficial organism could be detrimental to the success of the biological control strategy. The sterile substrate proved somehow difficult to address using same methods described for soil cultivation. The methods should be further refined by thoroughly research projects following the entire vegetation cycle and more efforts should be given to the bioactivity of the plants at the root and leaf level, not just the morphological features and yield of the crops.

\section{Acknowledgements}

This work was supported by the German Federal Environmental Foundation, through a Ph.D. Scholarship (R. M. Giurgiu).

\section{References}

Abdelrahman M, Abdel-Motaal F, El-Saved M, Jogaiah S, Shigyo M, Ito S, Tran LS (2016). Dissection of Trichoderma longibrachiatum-induced defense in onion (Allium cepa $\mathrm{L}$.) against Fusarium oxysporum $\mathrm{f}$.Sp. Cepa by target metabolite profiling. Plant Science 245:128-138.

Althaf HSK, Srinivas P (2013). Association of arbuscular mycorrhizal fungi and other rhizosphere microbes with different medicinal plants. Research Journal of Biotechnology 8(6):2428.

Atkinson NJ, Urwin PE (2012). The interaction of plant biotic and abiotic stresses: from genes to field. Research Journal of Biotechnology 8(6):24 28.

BahraminejadS, AbbasiS, Amiri R(2015). The effect of some medicinal and ornamental plant extracts against Fusarium oxysporum. Journal of Crop Protection 4(2):189-197.

Berges MSL, Hera C, Sulyok M, Schäfer K, Capilla J, Guarro J, Di Pietro A (2013). The velvet complex governs mycotoxin production and virulence of Fusarium oxysporum on plant and mammalian hosts. Molecular Microbiology 86(1):49-65.

Best NB, Hartwig T, Budka JS, Bishop BJ, Brown E, Potluri DP, ... Schulz B (2014). Soilless plant growth media influence the efficacy of phytohormones and phytohormone inhibitors. PloS ONE 9(12):e107689.

Bona E, Cantamessa S, Massa N, Manassero P, Marsano F, Copetta A, ... Berta G (2017). Arbuscular mycorrhizal fungi and plant growthpromoting Pseudomonads improve yield, quality and nutritional value of tomato: a field study. Mycorrhiza 27(1):1-11.

Chen F, Tang Y-N, Shen M-Y (2011). Coordination control of greenhouse environmental factors. International Journal of Automation and Computing 8(2):147-153.

Cornejo HAC, RodriguezLM, Vergara AG, BucioJL(2015). Trichoderma modulates stomatal aperture and leaf transpiration through an abscisic acid-dependant mechanism in Arabidopsis. Journal of Plant Growth Regulations 34(2): $425-434$
Di X, Takken FLW, Tintor N (2016). How phytohormones shape interactions between plants and the soil-borne fungus Fusarium oxysporum. Frontiers in Plant Science 7:170.

DingJ, Zhang Y, Zhang H, Li X, Sun Z, LiaoY, Xia X, Zhao Y, Shi K, Yu J (2014). Effects of Fusarium oxysporum on rhizosphere microbial communities of two cucumber genotypes with contrasting Fusarium wilt resistance under hydroponic conditions. European Journal of Plant Pathology 32(2):125-135.

El_Komy MH, Saleh AA, Eranthodi A, Molan YY (2015). Characterization of novel Trichoderma asperellum isolates to select effective biocontrol agents against tomato Fusarium wilt. The Plant PathologyJournal 31(1):50-60.

Elsharkawy MM, Shimizu M, Takahashi H, Ozaki K, Hyakumachi M (2013). Induction of systematic resistance against Cucumbermosaic virus in Arabidopsis thaliana by Trichoderma asperellum SKT-1. The Plant PathologyJournal 29(2):193-200.

Ganwar M, Dogra S, Gupta UP, Kharwar RN (2014). Diversity and biopotential of endophytic actinomycetes from three medicinal plants in India. African Journal of Microbiology Research 8(2):184-191.

Gawehns F, Houterman PM, Ichou FA, Michielse CB, Hijdra M, Cornelissen BJC, Rep M, Takken FLW (2013). The Fusarium oxysporum effector six6 contributes to virulence and suppresses i-2mediated cell death. Molecular Plant-Microbe Interactions 27(4):336348.

George S (2014). Monitoring occurrence and relative levels of rhizosphere microorganism on rockwool tomato crops across 2012/2013 growing season. MRes thesis, University of Nottingham.

Hadi M, Kashefi B, Sobhanipur A, Rezaarabsorkhi M (2013). Study on effect of some medicinal plant extracts on growth and spore germination of Fusarium oxysporum schlecht. in vitro. American-Eurasian Journal of Agriculture and Environmental Science 13(4):581-588.

Hajieghrari B, Mohammadi M (2016). Growth-promoting activity of indigenous Trichoderma isolates on wheat seed germination, seedling growth and yield. Australian Journal ofCropScience 10(9):1339-1347.

Hartmann T (2007). From waste products to ecochemicals: fifty years research of plant secondary metabolism. Phytochemistry 68(22):28312846.

Jelinski NA, Broz K, Jonkers W, Ma L-J, Kistler HC (2017). Effector gene suites in some soil isolates of Fusarium oxysporum are not sufficient predictors, of vascular wilt in tomato. Phytopathology 107(7):842-851.

Kahlen K, Strützel H (2011). Modelling photo-modulated internode elongation in growing glasshouse cucumber canopies. New Phytologist 190:697-708.

Kowalczyk K, Mirgos M, Bączek K, Niedzińska M, Gajewski M (2016). Effect of different growing media in hydroponic culture on the yield and biological quality of lettuce (Lactuca sativa var. capitata). Acta Horticulturae 1142:10-110.

Krupa P, Bandurska K, Berdowska A, Myga-Nowak M, Marczak M, Godela A, Bednarek S (2016). Improving the nutritional values of plant products through the use of biological agents such as Trichoderma viride in tomato plantations. Journal of Animal and Plant Sciences 23(3):3670-3676. 
Lace B, Genre A, Woo S, Faccio A, Lorito M, Bonfante P (2014). Gate crashing arbuscular mycorrhizas: in vivo imaging shows the extensive colonization of both symbionts by Trichoderma atroviride. Environmental Microbiology Reports 7(1):6477.

Lingyun WU, Dongwei YAO, Ming LI (2017). Effects of solid matrix priming with Trichoderma harzianum on seed germination, seedling emergence and photosynthetic capacity of eggplant. African Journal of Biotechnology 16(14):699-703.

López-Bucio J, Pelagio-Flores R, Herrera-Estrella A (2015). Trichoderma as biostimulant: exploiting the multilevel properties of a plant beneficial fungus. Scientia Horticulturae 196:109-123.

López A, Fenoll J, Hellín P, Flores P (2014). Cultivation approach for comparing the nutritional quality of two pepper cultivars grown under different agricultural regimes. LWT-Food Science and Technology 58(1):299-305.

Martinez FD, Santos M, Carretero F, Marin F (2015). Trichoderma saturnisporum, a new biological control agent. Journal of the Science of Food and Agriculture 96(6):19341944.

Michałojć Z, Jarosz Z, Pitura K, Dzida K (2015). Effect of mycorrhizal colonization and nutrient solutions concentration on the yielding and chemical composition of tomato grown in rockwool and straw medium. Acta Scientiarum Polonorum Hortorum Cultus 14(6):1527.

Ming Q, Su C, Zheng C, Jia M, Zhang Q, Zhang H, ... Qin L (2013). Elicitors from the endophytic fungus Trichoderma atroviride promote Salvia miltiorrbiza hairy root growth and tanshinone biosynthesis. Journal of Experimental Botany 64(18):5687-5694.

Musharof HM (2011). Therapeutic orchids: Traditional uses and recent advances-An overview. Fitoterapia 82(2):102-140.

Nieto-Jacobo MF, Steyaert JM, Salazar-Badillo F. B, Nguyen DV, Rostás M, Braithwaite M, ... Mendoza-Mendoza A (2017). Environmental growth conditions of Trichoderma spp. affects indole acetic acid derivatives, volatile organic compounds, and plant growth promotion. Frontiers in Plant Science 8: 102.
Ommati F, Zaker M (2012). Evaluation of some Trichoderma isolates for biological control of potato wilt disease (Fusarium oxysporum) under laboratory and greenhouse conditions. Journal of Crop Protection 1(4):279-286.

Postma J, Clematis F, Nijhus Eh, Someus E (2013). Efficacy of four phosphate-mobilizing bacteria applied with an animal bone charcoal formulation in controlling ur phosphate-mobilizing bacteria applied with an animal bone charcoal formulation in controlling Pythium aphanidermatum and Fusarium oxysporum f.sp. radicis lycopersici in tomato. Biological Control 67:284-291.

Rejeb IB, Pastor V, Mauch-Mani B (2014). Plant responses to simultaneous biotic and abiotic stress: molecular mechanisms. Plants 3(4):458-475.

Rubio MB, Hermosa R, Vicente R, Gómez-Acosta FA, Morcuende R, Monte E, Bettiol W (2017). The combination of Trichoderma harzianum and chemical fertilization leads to the deregulation of phytohormone networking, preventing the adaptive responses of tomato plants to salt stress. Frontiers in Plant Science 8:294.

Sundaramoorthy S, Balabaskar P(2013). Biocontrol efficacy of Trichoderma spp. against wilt of tomato caused by Fusarium oxysporum f. sp. lycopersici. Journal of Applied Biology \& Biotechnology 1(3):36-40.

Sheridan C, Depuydt P, De Ro M, Petit C, Van Gysegem E, Delaere P, ... Paradiso R (2017). Microbial community dynamics and response to plant growth-promoting microorganisms in the rhizosphere of four common food crops cultivated in hydroponics. Microbial Ecology $73(2): 378-393$.

Tremblay N (2017). Confronting the challenges of big data for precision agriculture. In: 2017 AAAS Annual Meeting (February 16-20, 2017), AAAS.

Zhang M, Xu J, Liu G, Yao X, Ren R, Yang X (2017). Protemic analysis of responsive root proteins of Fusarium oxysporum - infected watermelon seedlings. Plant and Soil 1-13.

Zubek S, Mielcarek S, Turnau K (2012). Hypericin and pseudohypericin concentrations of a valuable medicinal plant Hypericum perforatum $\mathrm{L}$. are enhanced by arbuscular mycorrhizal fungi. Mycorrhiza 22:149-152. 